\title{
La bactérie Vibrio cholerae lyse les bactéries environnantes et assimile leur ADN qu'elle intègre dans son propre génome
}

\author{
Marion Cannac, Thomas Petit
}

> Pour survivre dans une niche écologique ou en coloniser une nouvelle, les bactéries doivent acquérir des caractéristiques qui leur confèrent des avantages dans cette niche. Chez les bactéries, ces caractéristiques peuvent être acquises de façon horizontale, entre individus non apparentés co-existants dans le même environnement, par ce que l'on nomme des transferts horizontaux de gènes (THG) [1]. Les THG sont cependant peu stables en général, car l'ADN n'est pas toujours intégré dans le génome bactérien ou conservé sous forme de plasmide de façon pérenne. De plus, leur bénéfice est variable selon la qualité de I'ADN incorporé, l'information portée et le locus d'insertion dans le génome : si l'ADN est dénaturé, qu'il ne porte pas une information utile pour la bactérie ou qu'il s'insère dans un gène vital pour la bactérie, le THG peut être délétère, voire non viable.

La transformation naturelle est un type de THG. Elle consiste, pour une bactérie compétente, à incorporer dans son génome de l'ADN capté dans le milieu [2]. Vibrio cholerae est une bactérie Gram-négative qui peut être pathogène pour l'Homme, mais qui vit en milieu naturel sur les exosquelettes chitineux du zooplancton d'eau douce [3]. La compétence de $V$. cholerae, c'est-à-dire sa capacité à acquérir une molécule d'ADN exogène par ses caractéristiques naturelles, est sous le contrôle de la chitine et de ses produits de dégradation, ainsi que sous celui de la densité cellulaire via le quorum sensing. Ces facteurs externes modulent l'activité du régulon com de compétence de la bactérie, qui corres- pond à un ensemble de gènes contrôlant la compétence, régulés par un gène commun. Ce gène régulateur commun est $q s t R$, codant un facteur de transcription du même nom qui agit directement sur le régulon com pour permettre à la bactérie d'intégrer naturellement de I'ADN du milieu. QstR est régulé conjointement par tfox, gène régulateur directement activé par la chitine et ses produits de dégradation [4], et par hapR, gène maître du quorum sensing [5].

Pourtant, l'activation du régulon com ne suffit pas à expliquer la transformation naturelle efficace de V. cholerae. Borgeaud et al. se sont alors questionnés sur l'implication d'autres acteurs dans la transformation de V. cholerae [6]. Cette bactérie présente un système de sécrétion de type 6 (SST6), une machinerie protéique largement répandue au sein des bactéries Gram-négatives, qui permet d'injecter des protéines, appelées effecteurs, à la fois dans des hôtes eucaryotes et procaryotes. Ce système s'apparente à une seringue et est équipé d'un fourreau contractile qui, lorsqu'il est activé, propulse un tube protéique qui vient transpercer la membrane de l'hôte, induisant la libération des effecteurs dans la cellule cible [7]. Les auteurs ont alors exploré un potentiel rôle du SST6 de $V$. cholerae dans le processus d'assimilation de I'ADN en vue d'une transformation naturelle.

\section{Le cluster de gènes du SST6 est activé} par les mêmes voies que celui des gènes de compétence

Les auteurs ont voulu vérifier si les facteurs influençant le régulon de compé-
École normale supérieure de Lyon, Département de biologie, Master biologie, Lyon, France.

marion.cannac@ens-lyon.fr thomas.petit@ens-lyon.fr

tence contrôlaient également l'expression des gènes du SST6. Pour cela, ils ont utilisé une souche de $V$. cholerae modifiée de manière à ce que tfox soit artificiellement induit en présence d'arabinose dans le milieu de croissance, ce qui permet d'étudier l'expression de différents gènes en fonction de l'activation artificielle ou non de tfox. Comme décrit précédemment, l'activation de tfox permet l'expression des gènes de compétence. De même, en accord avec I'hypothèse des auteurs, les résultats de ces expériences montrent que les gènes du cluster codant le SST6 (vipA, vipB, vas $D$ et vask) ne sont activés qu'en présence d'arabinose, leur expression est donc sous la dépendance de Tfox.

Par ailleurs, les auteurs ont voulu tester I'importance des gènes hapR et qstR dans l'activation du cluster de gènes codant le SST6. Les auteurs ont donc évalué l'expression des gènes du SST6 lorsque tfox était activé mais que hapR et qst $R$ étaient inactivés. Ils ont alors mis en évidence la nécessité de l'activation de tfox mais également d'hap $R$ et de qst $R$ pour l'expression des gènes du cluster codant le SST6. Ainsi, comme pour le régulon com, le cluster codant le SST6 est activé par Tfox et HapR via l'activation du facteur de transcription QstR.

Le SST6 est induit par la croissance de la bactérie sur une surface de chitine semblable à son environnement naturel On sait donc d'une part que tfox est induit après croissance de $V$. chole- 


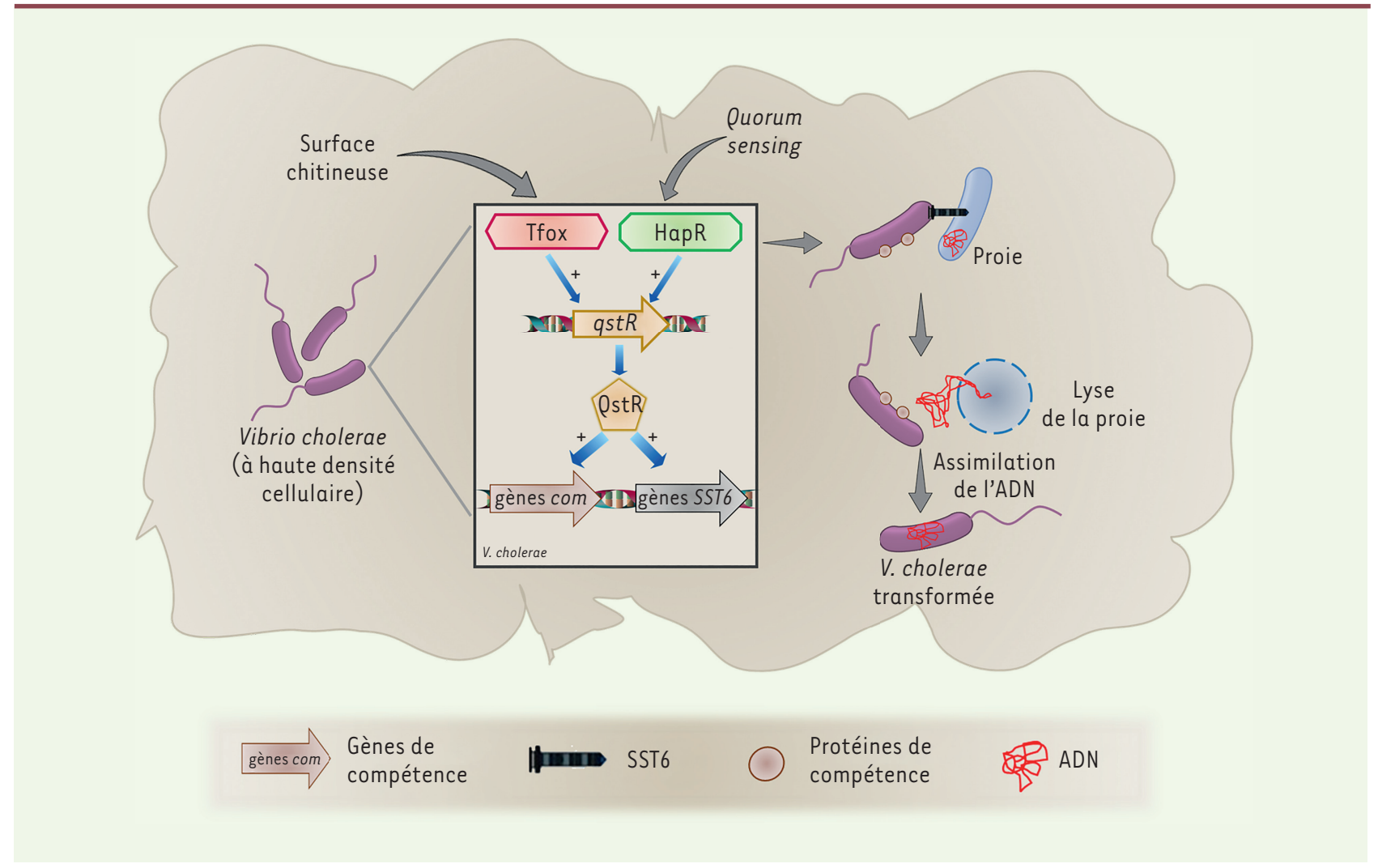

Figure 1. Le transfert horizontal de gènes est favorisé par le SST6 chez Vibrio cholerae. Le transfert horizontal de gènes est favorisé par la corégulation du régulon com des gènes de compétence et du SST6, contrôlée par le facteur de transcription QstR. Ce dernier est lui-même régulé conjointement par tfox, gène régulateur de la transformation, activé par le milieu chitineux, et hap $R$, gène maître du quorum sensing, activé à haute densité cellulaire.

rae sur la chitine et d'autre part que l'activation de tfox, avec le concours de hapR, permet l'induction via QstR des gènes du cluster codant le SST6. Afin de lier définitivement la chitine à I'expression du SST6, les auteurs ont ensemencé des bactéries exprimant VipA (une des protéines du SST6) fusionnée à la GFP sur un milieu chitineux ou non. En présence de chitine, ils ont alors observé l'expression de la GFP, sous forme de foci allongés, témoignant de l'expression des gènes codant pour le SST6. La bactérie V. cholerae assemble donc son SST6 lors de sa croissance sur un milieu chitineux naturel, environnement dans lequel la bactérie acquiert une compétence naturelle efficace. Ces résultats suggèrent que le SST6 pourrait jouer un rôle dans la compétence de la bactérie.
L'expression du SST6 en parallèle des gènes de compétence mène à la lyse des bactéries voisines et à la transformation naturelle de V. cholerae

Les auteurs ont alors émis l'hypothèse que le SST6 pourrait contribuer à la transformation naturelle de $V$. cholerae, certainement en facilitant la récupération d'ADN. Ils ont d'abord observé, par des essais de compétition, que l'activation de Tfox menait à une lyse significative des bactéries entourant $V$. cholerae, de manière dépendante des gènes hap $R$, $q s t R$, et ceux codant pour le SST6. Ces résultats suggèrent donc que le SST6 pourrait être impliqué dans la transformation en lysant les bactéries voisines de V. cholerae. Afin de tester cette hypothèse, des essais de transformation ont été menés entre deux souches de V. cholerae sensibles à l'action du SST6.
La première, désignée comme la souche proie, contenait dans son génome une cassette de résistance à la kanamycine et avait subi une inactivation de son SST6, l'empêchant de se comporter en «attaquante ». La deuxième, la souche prédatrice, jouissait d'un SST6 totalement fonctionnel et contenait une cassette de résistance à la rifampicine. La combinaison de ces deux résistances permettait de sélectionner uniquement les souches prédatrices ayant récupéré par transformation la cassette de résistance à la kanamycine de la souche proie. Les seuls essais dans lesquels était observée l'apparition de colonies bactériennes sur les milieux de double sélection, c'est-à-dire de colonies transformées, étaient ceux où Tfox était activé par l'arabinose et où à la fois le gène maître du quorum sensing hapR, le gène régulateur qst $R$, les gènes de 
compétence et ceux du cluster codant le SST6 étaient fonctionnels. Le SST6 de V. cholerae est donc bien nécessaire à la transformation naturelle de la bactérie, lorsque celle-ci croît sur un milieu chitineux à une densité bactérienne importante, par son action de lyse des bactéries voisines suivie d'une récupération de leur ADN.

\section{Conclusion}

Pour résumer, dans son environnement naturel, V. cholerae croît sur des surfaces chitineuses qui induisent l'expression de tfox. De plus, elle forme facilement des communautés de microorganismes appelées biofilms qui se composent de plusieurs espèces de bactéries. Dans ces biofilms, V. cholerae se trouve à haute densité cellulaire, ce qui induit l'expression du gène hapR. Les résultats exposés ici montrent que les protéines Tfox et HapR activent l'expression du facteur de transcription QstR qui, à son tour, active non seulement le programme de compétence, mais aussi les gènes codant le SST6 (Figure 1). Ainsi, V. cholerae peut induire la lyse de bactéries compétitrices via son SST6, provoquant la libération d'ADN dans le milieu. Puisque la bactérie exprime des protéines de compétence, elle peut facilement assimiler I'ADN environnant, ce qui permet sa transformation. Les bactéries appartenant à la même souche ne sont pas affectées par l'effet toxique du SST6 et des effecteurs qu'il permet d'injecter, car elles expriment des protéines inactivant ces effecteurs [8].

Les transferts horizontaux de gènes sont primordiaux pour l'évolution des bactéries. En effet, en assimilant I'ADN de microorganismes présents dans l'environnement, les bactéries peuvent acquérir de nouveaux gènes leur conférant un avantage sélectif. Le système décrit chez $V$. cholerae est en ce sens très efficace. $\varepsilon n$ effet, suite à la lyse par le SST6 de bactéries avoisinantes, I'ADN récupéré par V. cholerae a une probabilité plus forte de porter des gènes permettant de mieux survivre dans les conditions spécifiques du milieu, puisqu'il provient de bactéries qui y sont adaptées. Par ailleurs, le fait que I'ADN provienne de bactéries dont la lyse a été déclenchée par $V$. cholerae limite le délai pendant lequel l'ADN reste libre dans le milieu, ce qui augmente sa qualité et sa probabilité d'intégration au sein du génome de $V$. cholerae.

Les avantages acquis par transformation naturelle peuvent favoriser la réplication de la bactérie chez des hôtes comme l'homme, à son détriment. En effet, l'acquisition d'îlots de pathogé- nicité et de résistance aux antibiotiques contribue à la virulence de la bactérie, qui provoque chaque année des épidémies souvent mortelles. $\diamond$

Vibrio cholerae lyses surrounding bacteria and assimilates their DNA into its own genome

\section{LIENS D'INTÉRÊT}

Les auteurs déclarent n'avoir aucun lien d'intérêt concernant les données publiées dans cet article.

\section{RéFÉRENCES}

1. Thomas CM, Nielsen KM. Mechanisms of, and barriers to, horizontal gene transfer between bacteria. Nat Rev Microbiol 2005 ; 3 : 711-21.

2. Chen I, Dubnau D. DNA uptake during bacterial transformation. Nat Rev Microbiol 2004 ; 2 : 241-9.

3. Finkelstein RA. Cholera, Vibrio cholerae 01 and 0139 , and other pathogenic vibrios. In : Baron S, ed. Medical microbiology. Galveston (TX) : University of Texas Medical Branch at Galveston, 1996.

4. Meibom KL, Blokesch M, Dolganov NA, et al. Chitin induces natural competence in Vibrio cholerae. Science $2005 ; 310$ : 1824-7.

5. Lo Scrudato M, Blokesch M. A transcriptional regulator linking quorum sensing and chitin induction to render Vibrio cholerae naturally transformable. Nucleic Acids Res 2013 ; 41 : 3644-58.

6. Borgeaud S, Metzger LC, Scrignari T, et al. The type VI secretion system of Vibrio cholerae fosters horizontal gene transfer. Science 2015 ; 347 : 63-7.

7. Ho BT, Dong TG, Mekalanos JJ. A view to a kill: the bacterial type 6 secretion system. Cell Host Microbe $2014 ; 15: 9-21$.

8. Dong TG, Ho BT, Yoder-Himes DR, et al. Identification of T6SS-dependent effector and immunity proteins by Tn-seq in Vibrio cholerae. Proc Natl Acad Sci USA $2013 ; 110: 2623-8$.

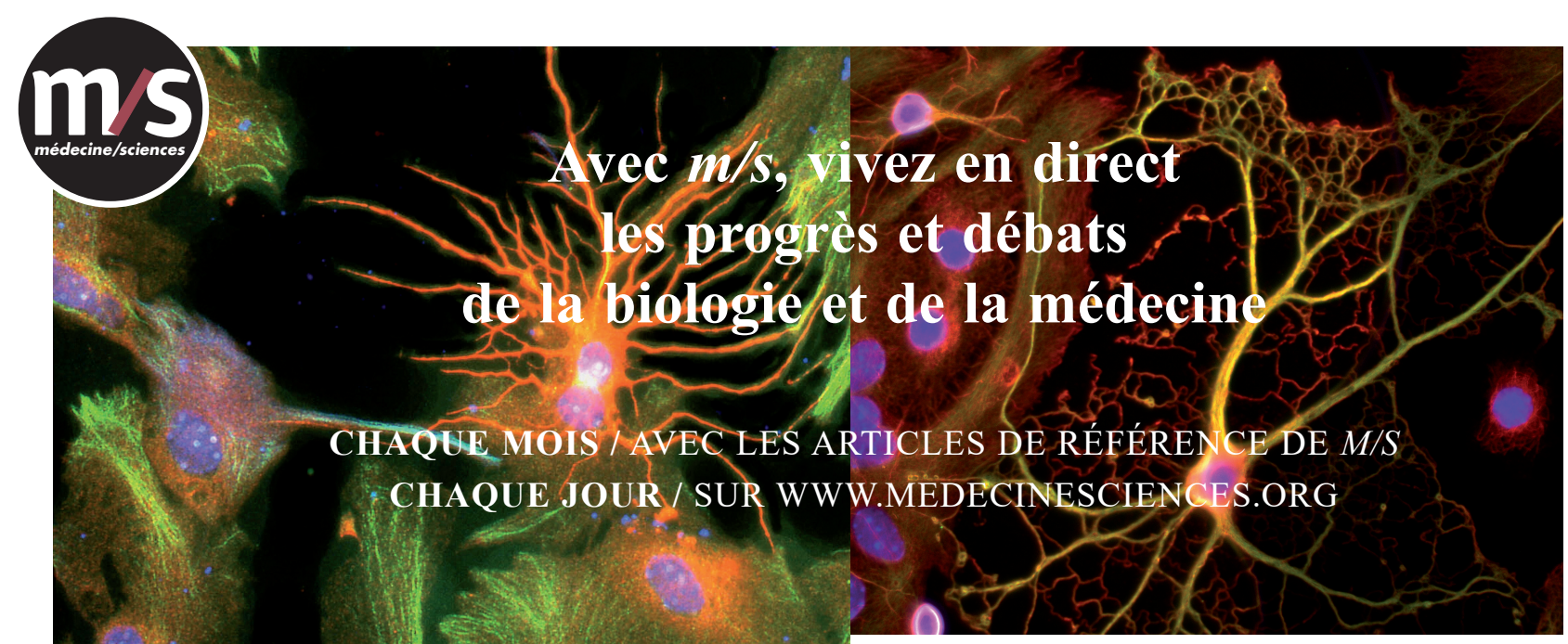

Abonnez-vous sur

WwW.medecinesciences.org 